\title{
Latest achievements of the negative ion beam test facility ELISE
}

\author{
B. Heinemann, U. Fantz, W. Kraus, D. Wünderlich, F. Bonomo, M. Froeschle, I. Mario, R. Nocentini, R. Riedl, \\ C. Wimmer
}

Max Planck Institute for Plasma Physics, Boltzmannstr. 2, 85748 Garching, Germany

The negative ion source test facility ELISE represents an important step in the European R\&D roadmap for the neutral beam injection (NBI) systems of ITER. Its aim is to consolidate the design and to gain early experimental experience with a large and modular Radio Frequency (RF) negative ion source and an ITER like extraction system of the same width but half the height of the ITER source $\left(0.9 \times 1 \mathrm{~m}^{2}\right)$. $\mathrm{H}^{-}$and $\mathrm{D}^{-}$beams can be extracted and accelerated up to $60 \mathrm{kV}$ for $10 \mathrm{~s}$ every $150 \mathrm{~s}$ out of the continuously operating plasma source.

For short plasma pulses $(10 \mathrm{~s})$, the extracted negative ion current densities in hydrogen have almost reached the ITER requirement $\left(329 \mathrm{~A} / \mathrm{m}^{2} \mathrm{H}^{-}, 286 \mathrm{~A} / \mathrm{m} 2 \mathrm{D}^{-}\right)$. Also the required long pulse source operation up to $1000 \mathrm{~s}$ (H-) / $3600 \mathrm{~s}$ (D-) could be demonstrated on ELISE with repetitive beam blips, but with reduced current densities. The main limitations are the amount and temporal stability of co-extracted electrons, especially in deuterium operation. This co-extracted electron current has to remain below the extracted ion current to avoid thermal overloading of the extraction grid. Magnetic field configurations, electric potentials of source surfaces close to the extraction system and caesium management are under investigation as tools for source performance optimization. Furthermore RF issues such as heating of source components, RF breakdowns and RF matching have been solved for high power source operation.

Keywords: Neutral Beam Injection, ITER, ELISE, negative ion source, radio frequency source

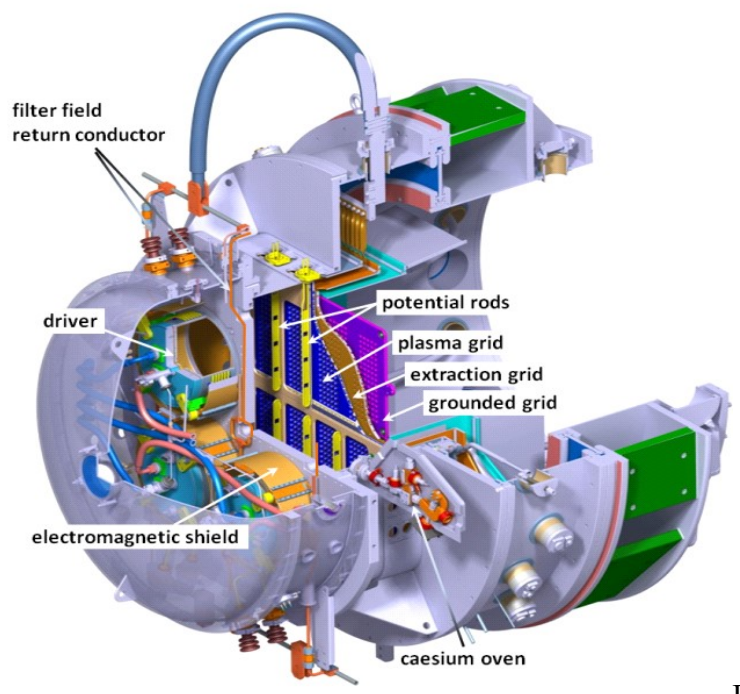

Figure 1: Overview of the ELISE ion source

\section{Introduction}

The test facility ELISE (Extraction from a Large Ion Source Experiment) is the first large RF (radio frequency) driven ion source for the production and extraction of negative ions. The ELISE ion source has with a size of $0.9 \times 1 \mathrm{~m}^{2}$ and an extraction area of $0.1 \mathrm{~m}^{2}$ the same width, but half the height of the ITER source. The design follows the ITER design as close as possible. However, some modifications have been implemented to allow for better diagnostic access and to improve the experimental flexibility. The aim is to better understand the open physics issues related to the size extrapolation from the prototype RF source $\left(0.3 \times 0.6 \mathrm{~m}^{2}\right)$ to the full ITER size $\left(0.9 \times 2.0 \mathrm{~m}^{2}\right)$. The plasma is generated in 4 cylindrical drivers via inductive coupling of the RF power to the plasma (two $1 \mathrm{MHz}$ RF generators, max power $90 \mathrm{~kW} /$ driver). The extraction system consists of a stack of three grids. Each grid is divided into two halfs (segments) and has 640 apertures totally with very similar aperture pattern and geometry as ITER (grouped in 8 arrays of $5 \times 16$ apertures, $\varnothing 14 \mathrm{~mm}$ ). Plasma operation of the source is feasible continuously, whereas beam extraction (up to $12 \mathrm{kV}$ ) and acceleration (up to a total voltage of $60 \mathrm{kV})$ is possible only in pulsed mode $(10 \mathrm{~s} / 150 \mathrm{~s})$ due to limitations of the HV-power supply. The negative ions are mainly produced by surface conversion of atoms and ions on the plasma grid (PG) surface which is 
covered by a thin caesium layer in order to reduce the work function. Therefore two caesium ovens (with a $1 \mathrm{~g}$ liquid Cs reservoir each) are installed on the source side walls and evaporate caesium into the source volume. Additionally, a magnetic filter field in front of the PG is created by a current of up to $5.3 \mathrm{kA}$ flowing vertically through the PG in order to reduce the co-extracted electron current. An overview of the ELISE test facility is shown in Figure 2, 2 and presented in detail in .

ELISE is equipped with a set of source diagnostics (pin probes, laser absorption spectroscopy and optical emission spectroscopy) and beam diagnostics : a tungsten wire calorimeter for visual beam monitoring, a diagnostic calorimeter with thermocouples, water calorimetry and IR imaging, and beam emission spectroscopy with 20 lines of sight.

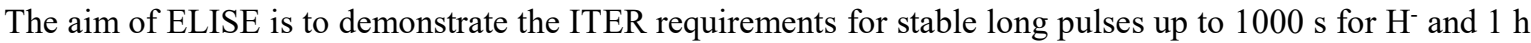
for $\mathrm{D}^{-}$, both at $0.3 \mathrm{~Pa}$ filling pressure: extracted current density of $286 \mathrm{~A} / \mathrm{m}^{2}\left(\mathrm{D}^{-}\right)$and $329 \mathrm{~A} / \mathrm{m}^{2}\left(\mathrm{H}^{-}\right)$, a ratio of co-extracted electrons to extracted ions below one and a beam non-uniformity of less than $10 \%$. After 4 years of experiments a remarkable progress could be achieved and important inputs for the design of the Neutral Beam Test Facility PRIMA in Padua and for the ITER NBI system could be provided.

\section{Results achieved}

First plasma was ignited at ELISE in February 2013 and routine operation for short pulses (20 s plasma with $10 \mathrm{~s}$ beam) was demonstrated up to current densities of $304 \mathrm{~A} / \mathrm{m}^{2}(\mathrm{H}) / 250 \mathrm{~A} / \mathrm{m}^{2}$ (D). Also long pulses up to $1 \mathrm{~h}$ ( $1 \mathrm{~h}$ source plasma with $10 \mathrm{~s}$ beam blips every $150 \mathrm{~s}$ ) could be performed, achieving $220 \mathrm{~A} / \mathrm{m}^{2}(\mathrm{H}, 1200 \mathrm{~s})$ and $155 \mathrm{~A} / \mathrm{m}^{2}(\mathrm{D}, 3600 \mathrm{~s}) / 193 \mathrm{~A} / \mathrm{m}^{2}(\mathrm{D}, 2700 \mathrm{~s})$.
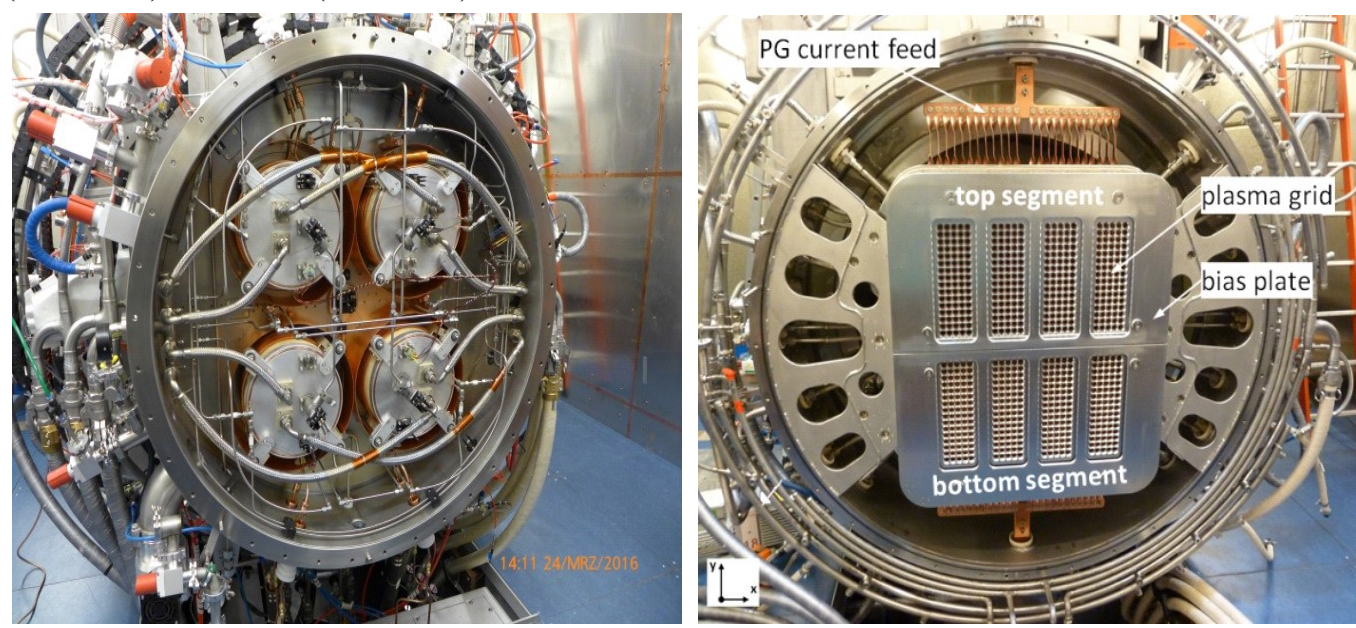

Figure 2: ELISE test facility, left: back side of ion source with 4 drivers (for operation in vacuum closed by a dome), right: view onto extraction system, showing the plasma grid and the bias plate; from the top the flexible copper conductors for the vertical PG current are visible, a corresponding connection is installed on the bottom side.

Achieving high ion currents for long pulses at high RF power is especially challenging because of the amount and temporal increase of the co-extracted electron current, in particular in deuterium operation. This can lead to thermal overloading of the second grid (extraction grid, EG) by impinging electrons. The electron current can be measured individually for each EG segment. These have been designed for a power load of $200 \mathrm{~kW}$ per segment, assuming equal co-extracted electron current at each aperture, but the operational limit is conservatively set at $125 \mathrm{~kW}$ (for specific cases at the ITER design value of $150 \mathrm{~kW}$ ) as the electron current might be non-uniformly distributed over the grid surface. Figure 3 presents examples of high extracted ion current densities for both isotopes, for long and short pulses at a source filling pressure of $0.3 \mathrm{~Pa}$. For these pulses the RF power has been adjusted to achieve high ion current with tolerable electron currents. Shown are the extracted ion current densities as well as the electron current densities on the top and the bottom EG grid segment. Left graphs (Figure 3) illustrate short pulses (20 s plasma phase with max. $9.5 \mathrm{~s}$ beam extraction). For both isotopes the negative ion current is quite stable over the pulse length, with some degradation in deuterium. For hydrogen also the electron currents on both grid segments are quite stable and almost symmetric; however, for deuterium the electron current on the top grid segment is much higher and increases steeply during the pulse. The pulse had to be terminated after $6 \mathrm{~s}$ in order to protect this segment from being overloaded. 


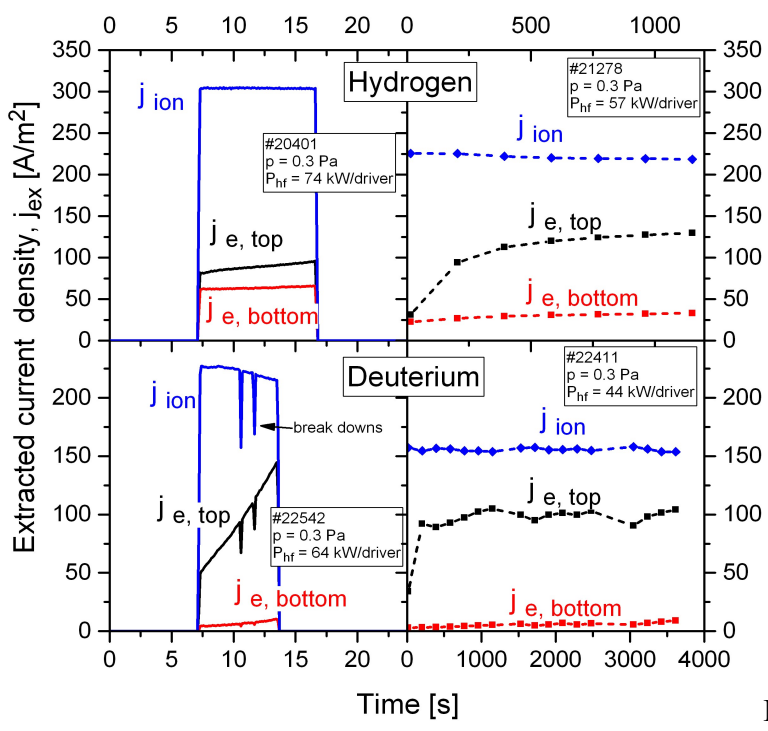
column long pulse plasma (1200 s H, 3600 s D) with pulsed extraction.

The right graphs in Figure 3 illustrate long pulses $\left(1200 \mathrm{~s} \mathrm{H}^{-} / 3600 \mathrm{~s} \mathrm{D}^{-}\right.$plasma phase with $10 \mathrm{~s}$ beam blips every $3 \mathrm{~min}$ ). For both isotopes the ion currents are again quite stable, slightly decreasing over time. The electron currents, however, are quite asymmetric and not stable over the long pulse duration. A very steep increase is observed between the first and second beam blip which is explained by caesium redistribution during the long pulse and caesium depletion on the plasma grid surface. Furthermore the currents are not stable during one beam blip (not shown here), indicating that caesium redistribution takes place also during the beam phase by backstreaming positive ions impinging on the source back plate. It has to be mentioned further, that long pulses with high performance cannot yet be done in a repetitive way, but need a careful preparation by short pulses in advance. The assumption is that the injected caesium has to be redistributed by plasma operation and short pulses in order to create proper conditions which assure a sufficient caesium flux - and therefore a low work function - on the plasma grid over the duration of the long pulse. Estimations about the Cs consumption for long pulse operation are presented in .
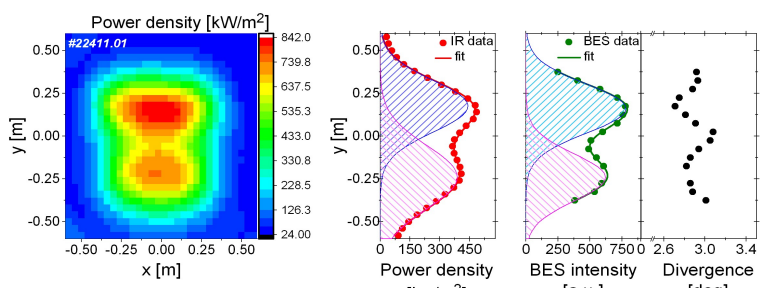

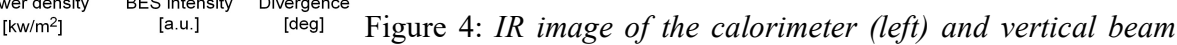
profiles evaluated from the IR image (center) and the BES (beam emission spectroscopy, right) for the top and bottom grid segment (\#24111, $39 \mathrm{~kW} /$ driver, $0.3 \mathrm{~Pa}, j_{D^{-}}=127 \mathrm{~A} / \mathrm{m}^{2}, U_{e x}=7.2 \mathrm{kV}, U_{a c c}=28 \mathrm{kV}$ ).

Up to now a simple and direct correlation between the top/bottom symmetry of the source plasma (caused by $\mathrm{E} \times \mathrm{B}$ drift) or electron currents and the top/bottom symmetry of the beam profile and divergence could not be found. The latter also seems to be influenced by the caesium and varies during the conditioning phase. For a well-conditioned source the calorimetrically measured ion current of each grid segment is quite similar, while the beam optic (measured by beam emission spectroscopy, BES) could be different. Figure 4 shows an example of the beam profile with an IR picture of the calorimeter (left) and the calculated profile evaluated from the IR image (center) and the BES data with the therefrom derived local divergence (right). The vertical beam profiles have been fitted with two Gaussians for each grid segment. The area under the two Gaussian fits (equivalent to ion current) is the same, while the average beam broadening (width of the Gaussians) is different.

In general, the applicable RF power and therefore the extracted negative ion current is limited mainly by the amount and temporal evolution of the co-extracted electrons. Measures to increase the ion current are usually accompanied by a higher level and steeper increase of the electron current. In order to achieve the ITER requirements the magnetic filter field strength and 3D topology are promising tools for the suppression of these co-extracted electrons. First experiments have been carried out and are described in section 3 .

Recently also promising experiments with "vertical potential rods" above the bias plate (Figure 1) have been started to influence the various potentials in front of it. By these rods it was possible to symmetrize and to reduce the co-extracted electron current. This again allowed to increase the RF power and by that the extracted ion current . Additionally performing long pulses at higher RF power was limited by several technical issues: (i) 
thermal overloading of different components in the vicinity of the drivers and (ii) electric breakdowns which occur randomly inside the vacuum environment around the drivers ("dome"), even at very low pressure of $10^{6}$ mbar. The mitigation activities are described in section 4 and 5 .

\section{Magnetic filter field optimization}

The geometry of the PG has been designed to achieve a uniform PG current density distribution across the width of the grid and hence a best possible uniformity of the filter field in front of it . Generally higher magnetic fields are required for deuterium operation than for hydrogen. Typical PG current values are $2.5 \mathrm{kA}$ for $\mathrm{H}$ and 4 $\mathrm{kA}$ for D operation; the system is installed for $5.3 \mathrm{kA}$, but designed for up to $8 \mathrm{kA}$. The filter field strength in the center of the PG corresponds to about $1 \mathrm{mT} / \mathrm{kA}$. Field strength and bias potential between PG and source wall interplay with each other and a compromise has to be found for each isotope to suppress the electrons sufficiently without reducing the ion current significantly.
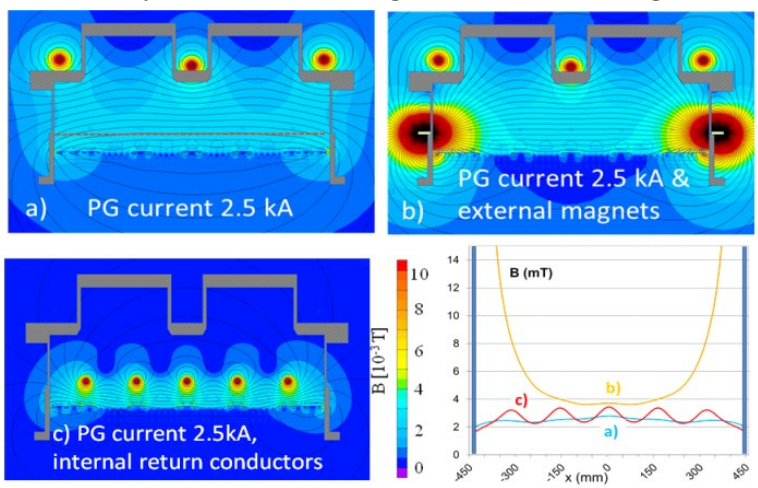

Figure 5: 2D magnetic field topology of ELISE (horizontal cross section), a) standard configuration, b) adding permanent magnets along lateral walls, c) internal return conductors, bottom right: horizontal profile across the $P G$.

The 3D topology of the filter field can be shaped either by adding permanent magnets along the lateral walls of the ion source and/or by modifying the position of the current return conductors. Figure 5 shows some possible configurations: a) the standard configuration with return conductors beside the drivers very close to the source back plate in order to reduce the field in the drivers (ITER like configuration). b) adding permanent magnets along the side walls; this does not significantly change the field strength in the grid center but produces a steep gradient at the edges. Three rows of CoSm magnets (cross section $9 \times 13 \mathrm{~mm}^{2}$ ) were positioned in strengthening as well as in weakening polarity with respect to the PG filter field . A significant improvement of the source performance for the strengthening configuration was observed, particularly for low pressure operation in $\mathrm{D}$ (Figure 6, ). The electrons are reduced by about 40\%, while the ions are affected much less and can be compensated by a slight increase of the RF power. c) internal return conductors in front of the PG with adjustable ratio between internal and external conductors; the magnetic field is compressed in front of the PG and plasma generation as well as expansion happen in a lowfield zone. This configuration is under preparation and can be combined with a) and b) in a flexible way.

\section{Mitigation activities against electric breakdowns around the drivers}

For ITER a RF power of $\sim 100 \mathrm{~kW} /$ driver is expected. But ELISE operation at RF power above 40 $60 \mathrm{~kW} /$ driver was technically prevented by RF-induced electric breakdowns which occured randomly in the driver area. The drivers of ELISE are placed inside a vacuum containment ("dome") to simulate the ITER conditions. This area is pumped independently from the source to a pressure as low as $10^{-6}$ mbar. Once a breakdown occurs, the pressure in the dome increases instantaneously due to gas release and the RF power is deposited outside the driver, while the internal plasma extinguishes. In the initial setup of ELISE the RF power switch-off was triggered by the plasma $\mathrm{H} \alpha$-light within typically $100 \mathrm{~ms}$ (due to the CODAC cycle time). To avoid damages of the ion source and RF generator a fast interlock $(<10 \mathrm{~ms})$ via a hardwareconnected photo diode was installed which is sensible to the breakdown light in the dome. 


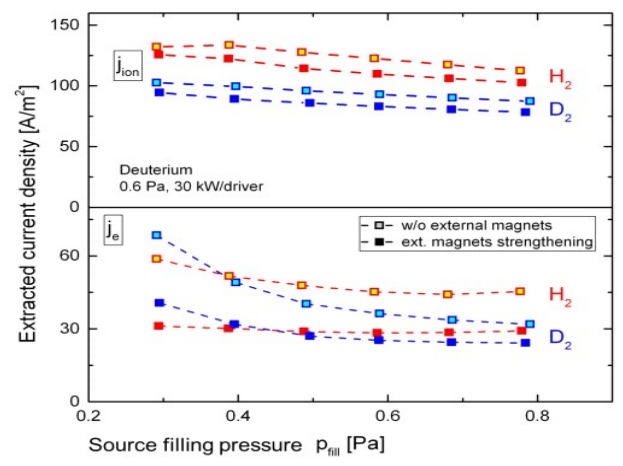

Figure 6: Performance improvement by adding external magnets to PG filter field in strengthening configuration, top: ion current density, bottom: co-extracted electron current density.

In order to further improve the situation and to avoid any dome breakdowns the following countermeasures have been considered:

\subsection{RF circuit}

The RF coils (6 turns) of two neighboring drivers are switched in series and connected to one RF generator. The intention of this circuit was to drive the same current through both coils and therefore generate similar plasmas in the two drivers. However, the RF voltage of both coils adds up to about $24 \mathrm{kV}$ (typically $2 \mathrm{kV} /$ turn). As they are electrically insulated from the source, the RF voltage will float around the source potential in a selfadjusting way. In order to reduce the RF voltage with respect to the source potential (by a factor of 2) two drivers have been switched in parallel to one generator. As a consequence the reduced inductance of the circuit has to be compensated by increasing the series capacitance from $\sim 1.5 \mathrm{nF}$ (variable) to $\sim 5 \mathrm{nF}$ which was achieved by adding $4 \mathrm{nF}$ fixed capacitors.

Operation in this configuration was possible up to $70 \mathrm{~kW} /$ driver without any breakdowns, but matching by adjusting the variable capacitor is difficult and the operation window is very narrow, because of the low frequency stability of the RF generators which are self-excited oscillators with $180 \mathrm{~kW}$ each ( $90 \mathrm{~kW} /$ driver). A further increase of the RF power was limited by either frequency flips or overcurrent in the RF generator.

An improvement for the RF power supply of the source was obtained by using two $150 \mathrm{~kW}$ solid state amplifiers instead of the free oscillators. The stable frequency of those amplifiers eases the matching to the plasma load. They are commercial radio transmitters which have been upgraded with an automatic frequency matching to minimize the reflected power and with a coupled power feedback control. These generators work at ELISE since beginning of 2017. The efficiency is much higher than that of the tube based oscillator $(90 \%$ instead of $60 \%$ ) so that water cooling is no more required .

\subsection{Reduction of electric field strength and avoiding triple points}

$\mathrm{RF}$ breakdowns in the dome area prevented to increase the RF power above $45 \mathrm{~kW} /$ driver and even forced a further power reduction over the experimental time. Visual inspection of the driver region during and after operation indicated that breakdowns have started at the RF line feedthroughs and directly at the coil windings. DC voltage holding tests and simple 2D-sensitivity analyses (using ANSYS) have been performed to better understand qualitatively the critical issues. The coils are manufactured from an annealed copper tube (Ø $8 \times 1 \mathrm{~mm}, \sim 7 \mathrm{~m}$ length) and do not have, due to manufacturing tolerances, a perfect line contact with the driver cylinder, but only a "several point" contact with unfavorable acute angles. At these triple points electron emission can start and trigger breakdowns. DC electric field strength calculations of this geometry in dependence of the gap between coil and driver showed that a reduction of the field strength is possible by (i) using quartz ( = 3.8) as material for the driver cylinder instead of $\mathrm{Al}_{2} \mathrm{O}_{3}(=9.5)$ for small gaps or (ii) increasing the gap between driver cylinder and coil (Figure 7).

Thus new driver cylinders were manufactured which combine both effects: quartz cylinders with identical inner diameter but reduced wall thickness $(6 \mathrm{~mm}$ instead the previous $8 \mathrm{~mm}$ ) provide a $2 \mathrm{~mm}$ gap between coil and driver cylinder. The floating position of the coil is assured via six "combs" between cylinder and coil and is therefore kept in the same distance to the plasma to insure identical RF coupling to the plasma. Combs made from a soft two-component silicone rubber material were manufactured which have a low relative permittivity $(\mathrm{r}=4)$ and a good flexibility to adjust to the geometric tolerances and to avoid small gaps to a large extent (Figure 8). The electric field strength near triple points in this area could be reduced by these measures by a factor of ten. Additionally one pair of copper coils has been coated by TEFLON ${ }^{\mathrm{TM}}$, an insulation method in use at positive ion RF sources on ASDEX Upgrade for many years. Since these modifications no further dome breakdowns occurred, no difference is visible between the copper coils and the TEFLON ${ }^{\mathrm{TM}}$ coated coils. The RF power upper limit is now only defined by the amount of co-extracted electrons. 
As the silicone comb fixation might not be applicable to ITER also a quartz driver with integrated quartz combs has been manufactured (Figure 8) and will be installed during the next maintenance period and subsequently tested.

\section{Cooling optimization of RF heated components}

In order to avoid mutual inductance between the neighboring drivers (which is suspected to be the cause of damages to the Faraday shields in previous experiments) each driver is shielded individually by an electromagnetic screen (EMS, a copper cylinder placed at $40 \mathrm{~mm}$ distance from the coil). From calorimetric measurements it was estimated that the EMS is heated by eddy currents of $\sim 1.4 \%$ of the RF power. In its initial configuration it was only cooled at two discrete positions around its circumference and the temperature increase (which induced a gas release and pressure increase) was limiting the applicable RF power or pulse length. A temperature of $350^{\circ} \mathrm{C}$ was set as operational limit, which induced several times a pulse termination, e.g. a pulse of $30 \mathrm{~kW} /$ driver had to be stopped after $1800 \mathrm{~s}$. Additionally, the support rods which fix the drivers to the source are positioned inside the EMS and also heated by eddy currents.

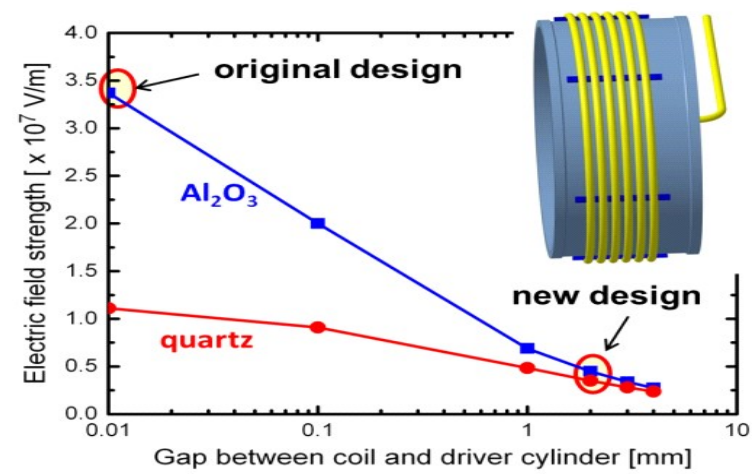

Figure 7: Electric field strength calculated for different gaps between coil and driver cylinder, for $\mathrm{Al}_{2} \mathrm{O}_{3}$ and quartz.

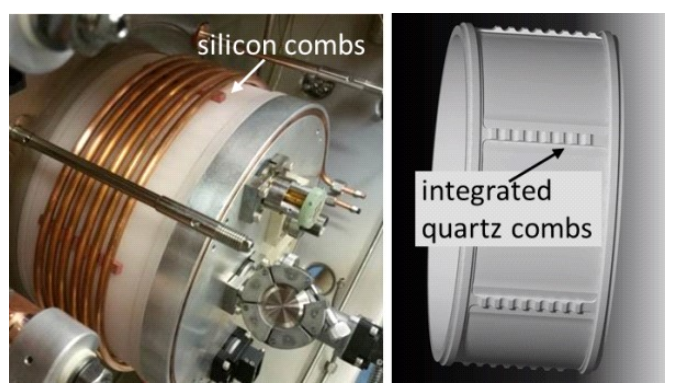

Figure 8: Quartz drivers, left: assembled with copper coil and silicon combs, right: with integrated quartz combs.

A new EMS design has therefore been developed and installed: the four support rods (per driver) have been replaced by actively cooled ones and the EMS is attached in 4 quadrants with good thermal contact to those rods. Heat conduction is further increased by increasing the EMS wall thickness from $1.5 \mathrm{~mm}$ to $4 \mathrm{~mm}$. Since the installation of the new EMS their temperature rise is no longer critical, e.g. $47 \mathrm{~kW} /$ driver results in an EMS temperature rise of $8^{\circ} \mathrm{C}$ which stabilizes after $250 \mathrm{~s}$ (Figure 9).

\section{Summary}

Within 4 years of operation the test facility ELISE has achieved remarkable progress with extracted current densities of $304 \mathrm{~A} / \mathrm{m}^{2}(\mathrm{H}) / 250 \mathrm{~A} / \mathrm{m}^{2}$ (D) for short pulses and $220 \mathrm{~A} / \mathrm{m}^{2}(\mathrm{H}) / 193 \mathrm{~A} / \mathrm{m}^{2}$ (D) for long pulses, both at $0.3 \mathrm{~Pa}$. It has to be noted that up to now these high current pulses required a careful caesium pre-conditioning and are not reliably reproducible. The limitation for a further increase of the ion current is given by the amount and temporal stability of the co-extracted electron current, which can also be strongly non-uniform between the top and bottom grid segment. Magnetic filter field variations by adding external magnets have illustrated possibilities for improvement and will be explored further with different positions of the PG current return conductors. Initial experiments with vertical potential rods above the bias plate improved the symmetry between the top and bottom grid segment and higher ion currents could be achieved. Both measures together with new ideas for improved Cs- distribution are considered as best knobs to achieve the ITER requirements in a repetitive way. 

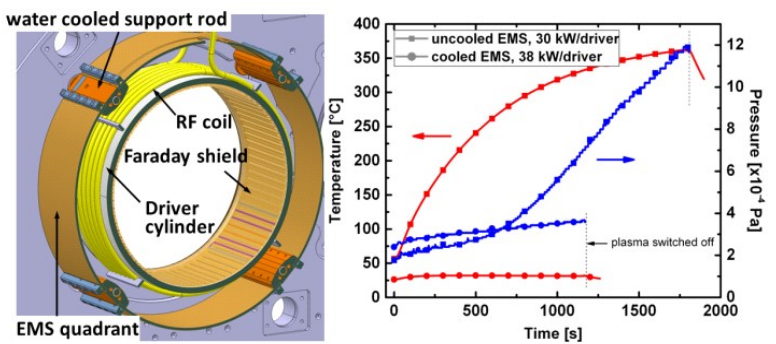

Figure 9: Electromagnetic screens of ELISE, left: design, right: temperature and pressure increase of old and new EMS.

The technical limitations of ELISE by RF-breakdowns in the dome region could be mitigated by using solid state amplifiers with fixed frequency and by a new driver design with quartz cylinders and a gap between RF coil and driver cylinder, which reduced the electric field strength by a factor of ten. The overheating of the electromagnetic screens could also be eliminated by an optimized design and is now no longer a limitation for the operation. 